\title{
Product Environmental Metrics for Printers
}

\author{
Jason Ord \\ Hewlett-Packard Company \\ 18110 SE 34th Street \\ Vancouver, WA 98683 \\ $+13602123191$ \\ jason.ord@hp.com \\ Ellen Chappell \\ Hewlett-Packard Company \\ 1000 NE Circle Blvd \\ Corvallis, OR 97330 \\ +15417155220 \\ ellen.chappell@hp.com
}

\author{
Scott Canonico \\ Hewlett-Packard Company \\ 1000 NE Circle Blvd \\ Corvallis, OR 97330 \\ +15417572000 \\ scott.canonico@hp.com
}

\author{
Tim Strecker \\ Hewlett-Packard Company \\ 1000 NE Circle Blvd \\ Corvallis, OR 97330 \\ +15417150305 \\ tim.strecker@hp.com
}

\begin{abstract}
Hewlett-Packard's Imaging \& Printing Group (IPG) is charting a course towards environmental leadership in its markets. To do this, IPG must look beyond just satisfying the regulations and identify opportunities for groundbreaking improvement. Carefully designed metrics are necessary to guide design, chart progress and set goals in this effort. IPG's Environmental Strategy Team is leading an initiative to establish these metrics internally. This paper describes the development process the authors followed to construct the initial metrics, which are focused on the "carbon footprint" of products under development. The paper also discusses the lessons learned developing the initial metrics, the results achieved thus far, implementation details, challenges, and future opportunities for improvement.
\end{abstract}

\section{Categories and Subject Descriptors}

J.2 [Computer Applications/Physical Sciences and Engineering/Engineering]

\section{General Terms}

Management, Measurement, Documentation, Performance, Design, Experimentation, Standardization, Verification

\section{Keywords}

Environmental product metrics, carbon footprint, environmental performance measurement, printers, printing

\section{Problem Statement}

\subsection{Motivation}

With a history of product stewardship and a Design for Environment (DfE) program, Hewlett-Packard's Imaging and Printing Group (IPG) has robust systems for ensuring its printing

Permission to make digital or hard copies of all or part of this work for personal or classroom use is granted without fee provided that copies are not made or distributed for profit or commercial advantage and that copies bear this notice and the full citation on the first page. To copy otherwise, or republish, to post on servers or to redistribute to lists, requires prior specific permission and/or a fee.

GreenMetrics'09, Month 6, 2009, Seattle, WA, USA.

Copyright 2009 ACM 1-58113-000-0/00/0004 ...\$5.00. products meet or exceed regulatory and market access requirements in worldwide markets. However, rapidly increasing customer environmental awareness and sophistication, as well as an internally acknowledged need to continuously reduce the environmental footprint of printing, necessitates a new array of standardized measurement systems. The metrics are necessary to facilitate establishment of new product and portfolio performance goals, enable design, track progress, and support communication to internal and external customers and stakeholders.

\subsection{Goals}

Ideal environmental performance metrics are scientifically rigorous and expressed in standard (e.g., SI) units. They should be broadly applicable, which in HP's case means they can be applied equally well to small appliance printers and commercial printing systems. Often these metrics must be normalized; i.e. expressed on a "per output basis" such as the environmental impact per unit of image printed. They must measure ill-defined aspects such as "recyclability" or life cycle carbon footprint. Finally, effective metrics should lead to succinct expressions of product performance that can be readily understood by the targeted user (which could span suppliers, designers and customers).

Our team of HP IPG engineers and scientists with both product development and environmental expertise is leading an effort to put into place a set of environmental metrics to guide product development and support external communication of product performance. The team's efforts to date include: an extensive benchmark of measurements systems used by other leading environmental manufacturers and competitors, selection and prioritization of environmental impacts and measures, and development of tools to enable accurate measurement given a reasonable amount of effort from R\&D and stewardship staff.

Our initial metrics were selected based on environmental impact, customer relevance, and stakeholder interest. They include measurements of a product's:

- Carbon footprint across its lifecycle;

- Use-phase energy consumption;

- Ease of recovery and/or recycling at the end of its use. 
Our team is aware that other important environmental impacts such as water usage, human toxicity, terrestrial and aquatic toxicity, etc. will not always track with the product's carbon footprint. However, our team has chosen to focus its early efforts on carbon footprint metric because 1) our internal design for environment processes already cover short-term tactics to address the other impacts, 2) it is an issue at the top of customers and management's minds, and 3) it is an incremental step in the right direction. If our team's metrics efforts successfully integrate a carbon footprint evaluation into HP's product development process, we will then move to include other key impacts in a second phase.

This paper describes the process our team used to develop our carbon and energy metrics along with our results thus far, our next-steps, and the challenges the authors see ahead.

\subsection{Carbon Footprint Notes}

A product's environmental lifecycle has several key phases, including:

- Manufacturing and supply chain

- $\quad$ Product use (supplies, energy consumption, media)

- $\quad$ End-of-life management

- Logistics in between all phases

A carbon footprint is an estimate of the greenhouse gas emissions associated with part or all of a product's lifecycle. The quality of a product carbon footprint figure can range from a crude estimate to one resulting from an expensive and detailed study for a particular product.

It is important to note that these estimates and studies are just models of the system carbon footprint. Like all models, carbon footprint models are only a convenient approximation of reality. The degree of accuracy with which the model represents a product's actual carbon footprint depends on the scope of the model, and the appropriateness and quality of its data and assumptions. There is no generally accepted definition of "proper" carbon footprint modeling procedures, although efforts to standardize this definition are underway, as detailed in the 3.2.2 "Validation" section of this paper.

Carbon footprints are typically expressed as a global warming potential (GWP) which implies that all the relevant greenhouse gas emissions have been accounted for (like methane and nitrous oxide) and that each gas's potency and persistency has been normalized against carbon dioxide for either a 20,100, or 500 year timeframe [1]. When the phrase "carbon footprint" or the label " $\mathrm{CO}_{2} \mathrm{e}$ " (carbon dioxide equivalent) appears in this paper, it refers to a GWP over a 100 year timeframe.

\section{SOLUTIONS}

Creating a set of metrics for all printer subsystems and lifecycle phases is a daunting task. For example, a recent internal environmental life cycle assessment for a small-business inkjet printer identified over 170 unique system-level parameters contributing to the product's overall environmental performance. Instead of addressing each and every parameter, IPG is taking the approach of identifying which ones are material for metrics based on their level of contribution. A key study that guided our analysis was the EuP Imaging Equipment Preparatory Study [2].

\subsection{Alternative Approaches}

During the beginning stages of our project, the authors completed a survey of existing systems to inform our efforts. Several of the notable evaluation protocols the authors encountered are summarized below.

GE's Ecomagination product review process uses life cycle assessments (LCAs) and business metrics as input to their product scorecard. This scorecard is then used to determine whether a particular product meets the company's environmental and operational performance objectives and, ultimately, makes it to market as an Ecomagination product. The environmental metrics that feed into the scorecard are energy (which includes carbon), chemical discharge, raw materials, and tradeoffs during use.

Other companies such as Herman Miller and Nike have developed their own internal environmental scorecard or metrics systems. Herman Miller has developed a Design for Environment (DfE) scorecard which covers material chemistry, recyclability, and design for disassembly. The DfE Score is a summation of these three metrics based on material weights. Both Herman Miller and Nike have spent considerable efforts in time and money to develop a mass-based supply chain system which includes material type to enable their scorecards. The IPG Environmental Strategy Team is applying best-practices observed in our review of these scoring systems as part of its metrics design process.

\subsection{IPG's Approach}

Once our team decided to focus our first efforts on establishing a set of printer GWP metrics, the team set about interviewing a sampling of the envisioned users in marketing, design, stewardship to understand what they wanted in a metric. The overwhelming advice given was to start small, establish a foothold, and build from early success. In particular, the team was cautioned that detailed product material data would be difficult to obtain in some cases, and that, although the objectives were widely acknowledged as important, technical staff capable of running the necessary analyses were already fully committed to other work.

The interview process identified two main user inquiries:

- Which of the two (or more) sets of materials I am considering for my subsystem has a lower carbon footprint, and how does the difference between the sets compare to the overall product footprint?

- What is the difference to the overall footprint when I change system level parameters such as print cartridge yield or power consumption during standby (particularly if I am not an expert in any of those areas)?

To satisfy these inquiries, the team defined the following project goal: Provide product designers (in the broadest possible sense of the word) with a business decision support tool that allows them to understand the carbon footprint implications of their decisions and the opportunities for improvement. 


\subsubsection{Metrics description}

In pursuit of the goal stated above, our team broke the printer and cartridge mass into 32 different materials. The team also developed an energy estimator that allows the user to change several key hardware and firmware parameters and compute a new lifecycle energy estimate. The team then linked all of this together into a "metrics tool" spreadsheet.

To use the metrics tool, the user must enter:

1. The masses for 32 unique materials (ranging in complexity from "steel" to "logic board assemblies") for the printer and all consumables, such as print cartridges.

2. The image yields for all consumables (ink and toner cartridges).

3. The expected life of the print system and the images printed per month (not to be confused with physical pages, as the two will be different when duplexing).

4. Whether or not the printer has a duplexer, and its speed.

5. The speed of the device, the power consumption levels for each of its modes and any wait times between modes.

When done, the user gets the following metrics in return:

1. An estimate for the total system lifecycle $\mathrm{CO}_{2} \mathrm{e}$, and a breakdown of such for the printer, consumables, paper, and electricity.

2. An estimate of system $\mathrm{CO}_{2} \mathrm{e}$ per image printed and a graph of where this result stands compared to other possible monthly image print levels.

\subsubsection{Metrics scope}

The scope of the results has several notable omissions- including ink, toner, assembly, and the logistics past the assembly factory gate. All of these were omitted in the spirit of achieving the design goal- to support business decisions. Based on the EuP printer studies [2] and others, the team believed that their contributions were not material to most decisions being made from the carbon footprint analysis at hand and thus the team was justified in not requiring developers or users from considering them. The team will monitor these omissions and should their reasons for omission no longer hold, the team will address the issue in an upcoming release of the metrics tool.

\subsubsection{Metrics modeling methodology}

Our team chose a "Process" life cycle assessment (LCA) based approach to model the GHG contributions for each print system component. Specifically, each component is modeled with secondary (i.e. "generic") data. Other options for modeling include a process approach using primary data, or an Economic Input-Output (EIO) approach. An EIO approach models components by assigning them to one of 500 industrial sectors and specifying their monetary costs. A publicly available dataset then converts these proxies into estimates for the low level environmental inputs and outputs associated with each component.

A process LCA model can have greater resolution but is time consuming to perform. An EIO model may yield lower resolution but can speed the analysis. Both approaches are often combined and this is called a Hybrid LCA. Our team chose the process modeling approach to give designers insight into design choices not possible with EIO, and chose to use secondary data because it appears that doing so will meet the project goal without the enormous expense of collecting primary data.

\subsubsection{Emissions factors- the keys to the metrics}

The metrics tool converts the electricity consumed by the printer into a carbon footprint via electricity emissions factors in the form of " $\mathrm{x} \mathrm{kg}$ of $\mathrm{CO}_{2} \mathrm{e}$ per $\mathrm{kWh}$ at the end user". The electricity emissions factors our metrics tool employs are from 2005 and are published by the World Resource Institute [3].

As per the process approach, the metrics tool converts the 32 printer and cartridge material masses, as well as paper, to a carbon footprint using life cycle inventory information from the ecoinvent database [4]. The ecoinvent material processes are converted into $\mathrm{CO}_{2} \mathrm{e}$ emissions factors using the IPCC 2007 characterization factors for the global warming potential of air emissions [1]. The end result is an emission factor in the form of "x kg of $\mathrm{CO}_{2}$ e per kg of material".

There are several important assumptions to note in the material emissions factors. First, the ecoinvent database boundary spans cradle to factory gate, and includes gathering of the raw materials, logistic, energy, and capital equipment associated with that item until it leaves its factory gate. Second, the metrics tool takes engineering material emissions factors for items like steel and also adds a process emission factor to cover stamping and injection molding. Third, electronics are modeled on a mass basis, and separated into wiring, logic boards, and power supply boards. Since the electronics can have emissions factors an order of magnitude higher than engineering materials, it remains to be seen if the team can meet the accuracy requirements implicit in our goal by handling electronics in this fashion. Fourth, the emissions factors are typically based on representative European processes that are removed both in a temporal and geographical way from the actual supply chain (typically located in Southeast Asia) our team is trying to model.

Finally, the handling of paper is critical to the results of our tool given its importance to the end result. Again, the team employs inventory models from the ecoinvent database run through the IPCC 2007 impact method to create emissions factors. At this time, our paper factors do not take into account either the net sequestration or emission of $\mathrm{CO}_{2}$ by the fiber source (forest), or the release of greenhouse gases at the end-of-life (from incineration, aerobic or anaerobic decomposition). Paper is an area of our metrics tool that is under constant improvement.

\section{IMPLEMENTATION}

The IPG environmental metrics effort will undergo a build/test/fix development loop throughout 2009 and will subsequently roll-out metrics beyond carbon footprint once feasibility is established. "Pilot" usage of the metrics tool is underway in each of IPG's major product lines.

\subsection{Infrastructure}

HP employs a Distributed Modeling Environment (DME) which is a web-based user interface for modeling applications including 
computational fluid dynamics and thermal analysis simulators. The environmental metrics tool uses this infrastructure to provide an easy to use graphical user interface. Material inputs based on mass are entered into the system (see Fig. 1) according to material type or subassembly.

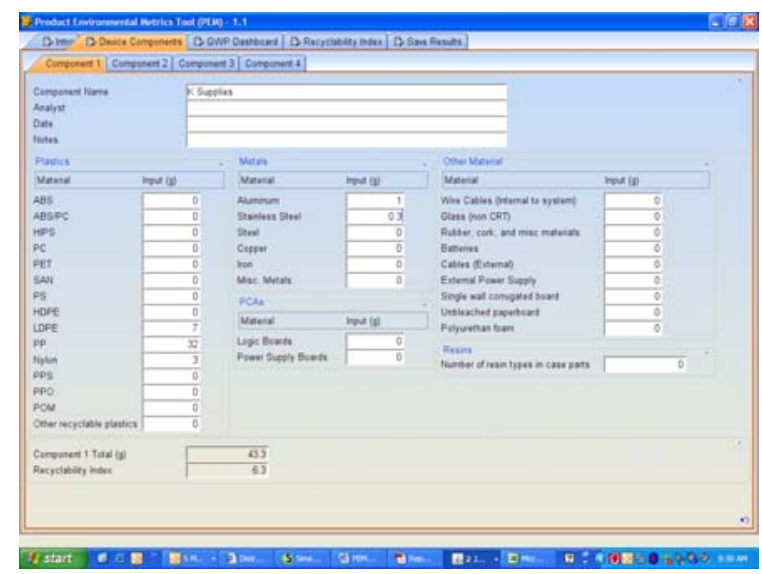

Figure 1, Metrics tool user interface

Figure 2 shows the "GWP Dashboard" which contains fields for the user to enter system level parameters such as printer life. This is also where output figures and graphs are shown, including a Pareto chart of carbon footprint by component, the carbon footprint per page versus lifetime images, and system carbon footprint versus lifetime images printed.

The DME interface simplifies and enriches the user experience with the tool. It also provides the data management solution for the metrics program. The DME system controls revisions and facilitates the sharing and leverage of models across IPG.

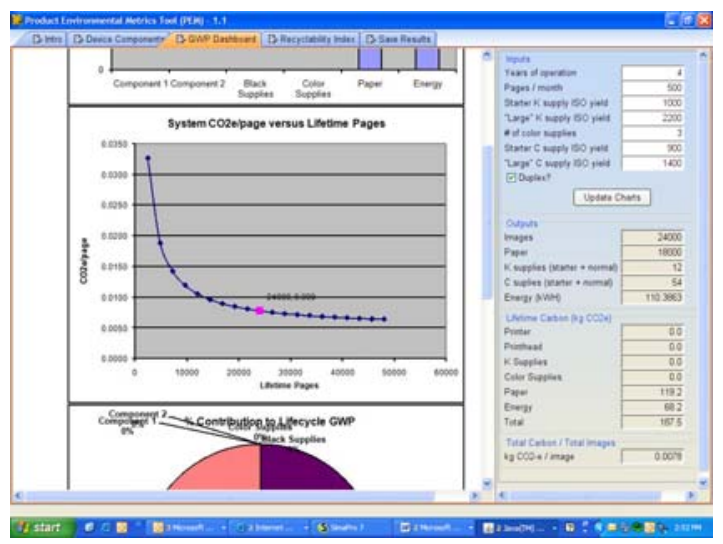

Figure 2, Metrics tool output page

\subsection{Challenges}

The challenges faced by even our initially carbon-focused metrics package are daunting and include:

- A carbon footprint is time and resource consuming to perform, due to gathering the required data (or building the data automation) and the significant amount of organizational comprehension necessary.

- A carbon footprint contains a large amount of uncertainty stemming from the data and assumptions employed by the model. A recent paper on the subject states that "LCA error margins can easily surpass $10 \%$ for energy and greenhouse gases" [5]. Given the complexity of printers and the "business decision enabling" goal for our scheme, the error of our method is certainly higher than $10 \%$.

The authors don't have enough room in this paper to fully address all of the key challenges faced, but do address the challenges of organizational adoption and validation below.

\subsubsection{Organizational Adoption}

No matter how well metrics are engineered, unless thought has been put into how they will be integrated into the design process and into the organizational culture, their adoption and impact will not be guaranteed. Along with providing tools, one must consider the goals for the metrics and the existing design processes.

The development team recognized early on that the metrics under development would need to be understood and used by both designers and stewards. Deliverables based on the metrics ultimately need to be built into the development process and overseen by the stewardship community. To start out the deliverables should be fairly easy to satisfy. In the first phase, a program team will only be expected to use the tool and document that they have done so. As more programs, use the tool and data is added to the database, this will generate a performance baseline as a second phase. Once the baseline is established, it will be possible to use it in a third phase to set new environmental performance goals that future teams will need to meet.

To facilitate this, the team developing the metrics had representatives from internal stakeholders, including Marketing, $\mathrm{R} \& \mathrm{D}$, and Operations, as well as high-level management sponsorship. The actual metrics themselves were designed to be science-based, but not so complicated as to require either in depth knowledge of assessment techniques or extensive hand holding on the part of the stewards.

The tool itself is not a "black box" where a user inputs data and gets the results back without any knowledge of how it was arrived at. Transparency is particularly important in an organization with a strong engineering culture. The vetting of the metrics tool was done to both validate the metrics themselves and to educate influential future users about them. In other words, the team wanted to create a "pull" and not just a "push" for organizational adoption.

Finally, the metrics tool needed to be highly accessible and not isolated from other tools that the design community uses on a regular basis. The inclusion of this tool within the DME system does just that. The development team also recognizes that utilization of the tool, especially the global warming potential and recyclability pieces, would require information that the engineers may not have ready access too today. Getting this information will need active engagement with the internal design and bill of material tools, the operations group, and with suppliers.

\subsubsection{Validation}

Our metrics team is keenly aware of the need to assess our first metrics package to ensure that it has the measurement fidelity necessary to achieve its stated goal: quantify environmental implications of product designs to support business decision 
making. In other words, what is the "signal to noise ratio" and is it acceptable?

The carbon footprint of a product is not a physical property that can be directly measured. Instead, it is a model that is built from many pieces of primary and estimated data, such as the $\mathrm{CO}_{2} \mathrm{e}$ emissions for the manufacture of a microprocessor. These pieces of data are then glued together with system level assumptions such as customer behavior. A tension then naturally arises between the level of modeling detail and the accuracy of the result. To help our metrics package find the right balance between these poles, the team is enlisting four tactics.

First, our team is conducting a life cycle assessment (LCA) of a product and comparing its results to those of the metrics package. This is essentially comparing the results of a full parameter set model to those of a reduced set model to make sure that the key parameters were retained and properly represented.

Second, our team is seeking out an external party to review our tool to make sure it achieves its goal. The team is currently engaging with the Carbon Trust, a UK government entity, to investigate the possibility of one of their agencies reviewing our model.

Third, our team is staying abreast of all relevant standards that exist or are emerging in this space. The existing ISO 14040 \& 14044 standards for Life Cycle Assessment give a basic foundation for all environmental metrics work, but are too broad to stand by themselves. The only major product carbon footprint standard in existence is PAS 2050 from the British Standards Institute but it is focused on external carbon labels instead of internal measurement. Other emerging product carbon standards include the World Resources Institute Scope 3/Product and ISO TC207 SC7.

Fourth and finally, our team is engaging a broad community of internal users and experts to help identify problems and improvement opportunities. To this end, the assumptions and calculations are highly transparent to the user. For example, an internal packaging expert can look at the materials $\mathrm{CO}_{2} \mathrm{e}$ emissions factors employed by the tool. If the expert felt that the factor for molded pulp cushions was too high, she could directly contact the metrics team or use a tool discussion forum to make her case for change.

\section{CONCLUSIONS}

IPG's efforts to develop new environmental metrics to assess life cycle environmental impacts of printing products has progressed through a number of project stages and delivered a prototype measurement tool now in pilot testing across the organization.

In addition to pilot testing, the team is fielding ad hoc requests for access to the tool and feedback on its use from an expanding cross section of the organization. The team is gaining confidence that it developed a workable, useful and technically rigorous set of metrics that will influence IPG's product design processes in the future.

The authors see three primary areas which the research community could help improve both internal and external environmental metrics systems.
First, the data upon which the metrics are built must be continuously improved and audited. "Improvement" means continuously closing the gap between what the data actually represents and what the data is intended to represent in its modeling context. This means making data available that is as close to the component being modeled in terms of supplier, location, and point in time as required by the modeling goal.

One emerging scheme that enables continuous data improvement and auditing is called "earthster" [www.earthster.org]. It provides an open-source approach for suppliers and manufacturers to rapidly share and vet product environmental data. The research community should identify what information needs to be shared, how it should be shared (what format and forums), and what the quality control process should be.

Second, the product category rules (PCRs) for IT industry products must be established, as called for in the PAS 2050 standard. The product category rules specify universal product usage models, study scope \& cut-off rules, and even the appropriate secondary data sets. The PCRs enable comparisons between similar products and cooperation between manufacturers, especially in regards to identifying issues common to the industry's supply base. The PCRs must be established collaboratively, including all the major stakeholdersmanufacturers, governments, NGO's, and academia, and must contain a strong ethic of continuous improvement.

Third, the authors see a need for an open-source set of set of scientifically-based environmental indices that effectively and credibly communicate the environmental performance of IT products both internally and externally. These indices should look beyond carbon to other key environmental impact categories, and may need to include impacts not traditionally included in LCA like "non-quantifiable environmental impacts such as biodiversity loss and site-specific impacts such as pollutant and noise emissions" [5].

\section{ACKNOWLEDGMENTS}

Our thanks to the entire HP IPG Product Environmental Metrics program team, and its sponsors and stakeholders; Stacey Wueste, Glen Hopkins, and Ayn Lavagnino.

\section{REFERENCES}

[1] Climate Change 2007. IPCC Fourth Assessment Report. The Physical Science Basis

[2] Stobbe, Lutz, et al. "EuP Preparatory Studies 'Imaging Equipment' (Lot 4)" Task 1 though 8 Final Reports. Fraunhofer IZM. Berlin, Germany. 2007 -2008

[3] Indirect $\mathrm{CO}_{2}$ Emissions from Purchased Electricity. Version 3.0. December 2007. Developed by World Resources Institute (WRI)

[4] ecoinvent Centre (2007), ecoinvent data v2.0. ecoinvent reports No.1-25, Swiss Centre for Life Cycle Inventories, Dübendorf, 2007

[5] Prakaash, Manhart, et.al. "Environmental product indicators and benchmarks in the context of environmental labels and declarations". Oko-Institut e.V. Brussels, Belgium. December 2008 